\title{
GRAMMATICALIZATION PATHS AND CHAOS: DETERMINISM AND UNPREDICTABILITY OF THE SEMANTIC DEVELOPMENT OF VERBAL CONSTRUCTIONS (PART 3 - CHAOS IN GRAMMATICALIZATION)
}

Keywords: grammaticalization paths, verbal semantics, Chaos Theory, Cognitive Linguistics, semantic maps

\begin{abstract}
This paper demonstrates that by applying Chaos Theory to the modelling of the evolution of verbal forms and verbal systems, it is possible to view classical grammaticalization paths as universal, and align this deterministic assumption with the unpredictability of concrete grammatical developments. The author argues that such an explanation is possible because traditional grammaticalization paths do not represent realistic cases of grammatical evolutions, but rather correspond to abstract and non-realistic deterministic laws which codify the order of the incorporation of new meanings to the semantic potential of a gram. Therefore, from a synchronic perspective, they can be used to represent the semantic potential of a form as a map or a state. In contrast, a realistic development emerges as a trajectory connecting such maps or states. Consequently, the cross-linguistic typological model of realistic evolutionary processes of a certain type corresponds to a state-space - it is a cluster of all possible trajectories the grams of a certain class can travel. This article - the last of the series - will formulate a chaotic model of the realistic evolution of verbal grams.
\end{abstract}

\section{Where we left off}

The previous papers of this series focused on the mathematical theory of chaos and its general applicability to other fields of science, including linguistics. In non-formal terms, chaos was defined as unpredictable behaviour of non-linear dynamic systems that, albeit governed by deterministic dynamic equations, are highly sensitive to 
initial conditions (for more formal treatment of chaos, see Devaney 1989; Strogatz 1994; Smith 1998). Chaotic systems typically exhibit properties such as (strange) attractors, bifurcations, basins, and emergence. As Chaos Theory is an abstract mathematical model it cannot be transposed to other branches of science in an unconstraint manner. On the contrary, in order to be successful, this transposition must be principled - that is, reductions, approximations and simplifications must be controlled. According to the definition proposed in the second paper of the series, a linguistic system can be viewed as chaotic if - after idealization and fictionalization - its model approximates the mathematical representation of chaos. That is, the robust features of the modelled grammatical system are compatible with properties exemplary of chaotic organizations mentioned above.

The application of Chaos Theory to linguistics, more exactly to the semantic evolution of verbal grams, began with the reinterpretation of a theory that is specifically concerned with language development - Path Theory. Path Theory is a conglomerate of views on the (mainly semantic) evolution of verbal grams (see Bybee, Perkins, Pagliuca 1994). In the previous paper, I argued that traditional paths are not models of realistic evolutions. While realistic grams accumulate senses during their evolution, paths formulate no prediction with regards to the extent of such an accumulation. Therefore, paths should rather be understood as abstract and theoretical principles governing evolutions of grams. At this abstract and theoretical level, where all the noise and friction are ignored and the system is profoundly idealized, paths can be viewed as universal and deterministic. While paths do not portray realistic evolutionary cases, they schematize sequences and possible ranges of senses incorporated into semantic potential of grams of certain types. As a result, they can be used as matrices, namely to represent semantic potentials of grams in a determined point in time. As a result, they can deliver models of synchronic states of grammatical constructions.

Having proposed an alternative view of the new epistemological status of paths, a question arose: How can we thus represent realistic evolutionary cases? The present paper - the last in the series - will provide a proposal of such an evolutionary model. I will argue that realistic evolutions are sequences of stages on state-space. In this state-space, each state corresponds to a map organized along a path-matrix (Section 2). This model will be demonstrated to exhibit robust features typical of chaos (Section 3). Consequently, this conceptualization of the grammatical life of verbal constructions will enable me to provide an explanation of all possible developmental cases, including the most anomalous and non-canonical ones and to align the deterministic nature of paths with the unpredictability of grammatical evolution. In the concluding section (Section 4) main results of this research be will presented and its limitations discussed.

\section{Realistic evolutionary model - trajectories of states}

Given that traditional paths epitomize the rules governing developments of grams (but not such developments themselves), a new manner of representing realistic 
evolutions of verbal constructions (that is, a representation that shows how grams actually change over a period of time) is necessitated.

Let us assume that in every point of time $t$, a gram is characterized by a set of properties which together constitute its state $a$. In the previous paper, it has been argued that by using standard paths, one can represent the semantic content of a gram as an amalgam of the values that have been acquired and preserved up to that particular moment in time. This set of senses has been accumulated in the order established by a given path and can thus be graphically organized by means of the template based upon that path. Consequently, the state of a verbal construction equals a semantic map (a network) of senses $x$ that have been stored in accordance with the universal laws, ranging from a certain value-stage $x_{\mathrm{a}}$ to another value-stage $x_{n}$. The realistic evolution of a gram corresponds to a modification of this map.

The modification can be qualitative and/or quantitative. The former type implies that the extent of a map changes - new senses are incorporated while older senses are lost. As maps can be more fine-grained and more coarse-grained, such qualitative modifications may be more microscopic or more macroscopic, respectively. This change of the qualitative range of a map can be illustrated by the evolution of the present perfect grams in Romance languages. In Modern Peninsular Spanish and in French of the $18^{\text {th }}$ and $19^{\text {th }}$ centuries, present perfect forms (pretérito perfecto and passé composé, respectively) are compatible with the senses of a resultative, experiential and indefinite perfect and with the sense of a hodiernal past. However, in certain Spanish dialects, the same gram has acquired the value of a hesternal past (yesterday's past) and/or a recent past (Bybee, Perkins, Pagliuca 1994). In Modern French, it further became compatible with the value of a general past (i.e. of any degree of remoteness; Grevisse 1975). This qualitative evolution can be represented in the following manner:

Stage 1

RESULTATIVE PROPER
INCLUSIVE PERFECT
RESULTATIVE PERFECT
EXPERIENTIAL PERFECT
INDEFINITE PERFECT
HODIERNAL PAST
HESTERNAL PAST
RECENT PAST
GENERAL PAST

Stage 2

RESULTATIVE PROPER
INCLUSIVE PERFECT
RESULTATIVE PERFECT
EXPERIENTIAL PERFECT
INDEFINITE PERFECT
HODIERNAL PAST
HESTERNAL PAST
RECENT PAST
GENERAL PAST

Stage 3

RESULTATIVE PROPER INCLUSIVE PERFECT RESULTATIVE PERFECT EXPERIENTIAL PERFECT INDEFINITE PERFECT HODIERNAL PAST HESTERNAL PAST RECENT PAST

GENERAL PAST

Figure 1. Qualitative development of maps

The other manner in which maps can be modified over time is quantitative. In order to understand this type of change, the concept of a quantitative map must be explained. A quantitative map is a semantic map that specifies not only the range of senses (i.e. the gram's polysemy) but also these senses' prototypicality. That is, even though a gram can be compatible with various senses, the extent of this compatibility is not identical for all such senses. Some senses are prototypical, while others 
are not. Although prototypicality is a complex matter (for a discussion see Evans, Green 2006; Gilquin 2006; Geeraerts 2010) its most immediate and visible indicator is frequency (Gilquin 2006; Gries 2006; Gries, Stefanowitsch 2006). Prototypical senses are common, whereas non-prototypical senses are rare. ${ }^{1}$

A simultaneous codification of such qualitative and quantitative information delivers a wave (Andrason, Visser 2015; Andrason 2016a, 2016b). In this model, the horizontal $x$ axis indicates the path with which the semantic potential of a form can be mapped (this corresponds to the qualitative mapping). The vertical $y$ axis indicates the degree of prototypicality (this range can be expressed in precise and absolute terms, i.e. from $0 \%$ to $100 \%$ or, in approximated and relative terms, for instance as void, non-prototypical, semi-prototypical, and prototypical). The correlation of the arguments of the qualitative $x$ axis with the values of the quantitative $y$ axis delivers the form of a wave (Andrason, Visser 2015; Andrason 2016a, 2016b).

Accordingly, the quantitative modification of a map refers to a change of the extent of prototypicality of each sense - a component of the map. This surfaces as a change of the form of a wave. For example, even though at three diachronic stages, a gram can be compatible with the same values, thus exhibiting the same semantic potential (i.e. the range of the $x$ axis is identical) the values of the $y$ axis fluctuate. As a result, the wave changes giving the impression of advancing along the path as if it was moving along a stream. Most importantly, the peak of a wave moves along the path, being located at different stages of it in different diachronic periods. This can be illustrated by the development of a hypothetical gram of an anterior-path type that gradually advances the position of its prototypicality peak from a more original stage to a more advanced stage (cf. Figure 2 below). Examples of this type of evolution in Semitic languages and Greek may be found in Andrason (2013a, 2014a) and Andrason, Locatell (2016).

Of course, a map can change both qualitatively and quantitatively at the same time. This will usually depend on the granularity of semantic distinctions adopted in an analysis. More coarse-grained maps usually fluctuate quantitatively, while in more fine-grained analyses, a qualitative change of the map will be observable more easily. The perception of a qualitative or a quantitative change also depends on the temporal interval that separates two measurement points. That is, if stage 2 is temporally close to stage 1 , quantitative modifications are more likely to be found. If the period separating two stages is extensive, qualitative modifications become highly probable to be detected.

A model of realistic evolutionary cases equivalent to Path Theory - i.e. model that would generalize over a number of developments in various languages - should encompass many instances of the modifications of maps. The intact preservation of the wave representation in such a model is virtually impossible, as the model would become illegible. In order to ascend to a higher level of generalization in which an elegant representation of the realistic evolution of grams could be posited,

Frequency itself is a complex concept and crude general distribution of senses in the semantic potential of a gram is only one of its possible aspects (compare Glyn 2010; Fischer 2010; Gries, Divjak 2010). 


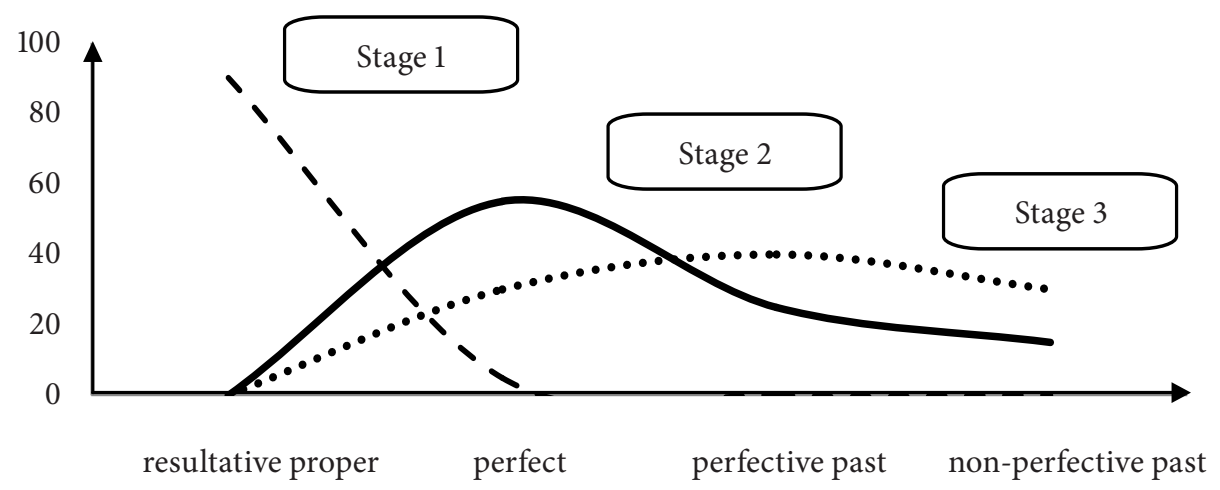

Figure 2. Quantitative development of maps ${ }^{2}$

further approximations are necessary. Most importantly, the state of a gram - i.e. its map or wave - must be represented in a manner that would allow to include in the representation a number of such states, ideally as many as possible of them.

This can be achieved if the state of a gram is represented as an abstract geometrical point, which numerically encapsulates the total behaviour of the gram in question. I will elaborate on this in more detail. As explained, the realistic evolution of a verbal construction will consist of the modifications of the states, i.e. it will correspond to an expansion or a shrinkage of the semantic map of this gram (in qualitative and quantitative terms). Since the state can be idealized and symbolized by a number or point, the total evolution will correspond to a sequence of such numerical states, thus delivering a trajectory of states. When a sufficiently large sample of evolutions of grams of a common taxonomical class has been represented in this manner, the model will yield a set of possible trajectories (paths of states). These trajectories (on which each point stands for a map or a wave) will show how states (i.e. semantic potential represented as senses connected by means of diachronic templates, which specify the order of acquisition of the values) change historically in various languages. The set of all states which the system may possibly achieve - i.e. the sum of all the trajectories of states found empirically - will yield the state-space of a given linguistic type or a category. This representation could determine all possible topological regions which the analyzed type of grammatical formations can visit - both those that are probable and those that are improbable although possible. Therefore, it could suggest exemplary modifications of the states and trajectories, i.e. modifications that are most likely crosslinguistically (for a more comprehensive discussion of the issue of state-space and its examples see Auyang 1998a, 1998b, 2000).

2 In this figure, the $x$ axis and thus the path template exhibit a coarse-grained form in which four stages are only distinguished. Most importantly, all the subtypes of a perfect have been grouped together under a single category and the various temporal variants of a past tense (hodiernal, hesternal, etc.) have been ignored (cf. the map in Figure $3(\mathrm{a})$ in the second paper of the series). The $y$ axis is numerical and corresponds to the precise degree of prototypicality based on frequency (i.e. from $0 \%$ to $100 \%$ ). 


\section{Chaos}

In the previous section, I formulated a model that could represent realistic evolutions of grams more adequately. As will be evident from the subsequent discussion, this model exhibits a prototypical chaotic behaviour - it fulfills the conditions established for chaos in non-mathematical systems and their representations. In other words, once the states of grams have been idealized and represented as points in a geometrical state-space, the model can be studied as for its own properties. These properties overall harmonize with certain robust features offered by prototypical chaotic systems.

First of all, the system modelled in this manner is evidently dynamic. The model represents the change of certain types of objects over time, specifying how the state of such entities is modified. In other words, since each stage on the trajectory and each point on the state-space represent the state of a gram at a given time $t$, the model determines the realistic evolution or the change of realistic states.

The model is also deterministic. Given that, within this new view, paths operate as deterministic laws governing the evolution of grams, like dynamic equations, they enable us to "calculate" the state of the construction at later, although not distant, points of development. Accordingly, since the life of a grammatical formation is controlled by deterministic rules, the equation determines a unique successor phase for every stage in the process. Namely, given certain initial conditions (the current state of a gram) and parameters (for instance, certain environmental factors), the rule - similar to a dynamic equation - predicts the gram's immediate subsequent behaviour. For instance, if an expression provides all the meanings typical of the category of a present perfect up to the experiential phase, one may assume that at the next evolutionary stage (and especially if the verbal system within which this form develops includes in its repertoire a gram whose prototypicality corresponds to more advanced sections of the anterior cline, e.g. past tense), the semantic potential of the gram will be analogous to the meaning offered presently, but additionally enriched by the subsequent sense predicted by the rule, i.e. the value of an indefinite perfect. As a result, one can, with a good degree of certainty, predict the short-term development of a given gram. It is highly probable that the gram's state will be modified in accordance with the rule predicted by the path, i.e. by incorporating or losing the values immediately adjacent to the extreme portions of the map (especially, if there is no environmental obstacle and/or if the adjacent environment allows it). The developmental process is deterministic within a short time interval, as each evolutionary stage that immediately follows must include one of the few possible extensions as predicted by the dynamic rule. It should, however, be emphasized that path-laws and state trajectories do not represent the same phenomena. While, as already explained, Path Theory's rules codify the direction and order of consecutively accumulated or developed meanings during the evolution of a category, the state trajectory of a gram represents consecutive sets of accumulated meanings. The former is entirely deterministic - it is a universal, abstract, theoretic law. The latter represents 
realistic evolutionary cases. Because it is driven by deterministic rules, it may be predicted. However, as in chaotic systems, this predictability operates within a short time span and within an error margin.

The deterministic nature of universal path-laws and a relative certainty as to the short-term development of concrete grams clashes with the long-term unpredictability of the future behaviour of grammatical formations. Although we control - to an extent - the state of the system at a given time $t$, and comprehend the deterministic nature of the governing rules, the exact state of a realistic grammatical construction after hundreds or thousands of years cannot be predicted, even within a margin of error. We cannot identify the set of properties the gram will reach after a long interval of time. This long-term unpredictability stems from the sensitivity to initial conditions. When determining the state of a gram, we establish a finite set of some features $f$ which are relevant to a given study. This will most often include the meanings developed and accumulated so far, as well as some salient, formal (phonological, morphological, syntactic and pragmatic) properties and the most relevant relationships (in all the above-mentioned aspects) the gram establishes with other elements of the language system. Even though we control a high number of features, there will always be something left out: some short-term, supposedly unimportant grammatical characteristics and relations. Furthermore, there is another set of elements (this time, extra-linguistic ones) such as sociological, cultural, economic, aesthetic or physical factors which can never be controlled entirely or even approximately. Many of them will be entirely absent in the depiction and modelling of the state of a gram. Accordingly, this state will always be represented as a finite number of data that necessarily disregards less relevant or secondary (according to the explainer's or experiencer's view) pieces of information. However, due to the infinite complexity of real-world systems, this ignored portion of information will invariably be immeasurable. It is physically impossible to determine all initial conditions - the network of such connections is too large to be manageable (Auyang 1998b: 344; Richardson, Cilliers, Lissack 2007: 33; Wagensberg 2007: 27, 56-60; Bishop 2011: 116-117, 121-123; Cilliers et al. 2013: 2). This reduction of infinite reality to a limited collection of features and the omission of (supposedly) unimportant properties and/or phenomena may be considered an error margin that is assumed in our description of the state. However, during the evolution of grams, this assumed inaccuracy (i.e. this minimal error corresponding to the approximation which has been assumed in determining the state) inflates exponentially. The smallest fluctuation of initial data affects the outcome of a process in such a drastic way that - although the deterministic laws operate at all stages and during the whole diachronic development - the predictability of the exact state of a gram after a long interval becomes highly uncertain, if not impossible. Thus, even the smallest divergence in initial or intermediate data will have an immense impact on the future shape of a construction after a sufficiently long time. In a similar vein, even the least significant differences in the state of two similar grams will cause these two objects, almost equal at the beginning, to acquire significantly different appearances after an extended period of time. This explains why a single mother tongue can give rise 
to languages that, after a time, often become highly different. There is always a difference in broadly understood initial conditions that are either acknowledged or ignored in the modelling. These controlled or non-controlled values are responsible for a subsequent divergence.

State trajectories of grams idealized in a model of the evolution of grams are also unrepeatable - they are a-periodic. Even though evolutions are driven by deterministic laws driving a formation from a pre-established input (an initial stage according to a path-law) into a pre-established output (a meaning which corresponds to the final stage of a path-law), concrete developmental processes, which are propelled by the same path-law, will never be identical. It is evident that there are no two identical languages developing from any two sources, be they highly distinct or extremely similar. The aperiodic character is assured by the error margin in the initial conditions and its inflation during the further development. For instance, the grammatical growth of two originally resultative formations in two distinct settings (different languages or different historical époques in a single language) will not be indistinguishable and entirely alike - the evolutions will never duplicate because the states of the grams will diverge in some values (Larsen-Freeman 1997; Bybee 2010: 198). No two perfects, perfectives or past tenses are identical - their states invariably differ in some features.

The terminal stage of the development as posited by Path Theory corresponds to a meaning which is the last to be incorporated and the last to be abandoned. It is the final value displayed by the gram before it disappears or is recycled for new grammatical purposes. In my model of the evolution of grams - built around the idea of the modification or trajectories of states - the terminal phase in the development of a gram equals the ultimate state of a formation, the final geometrical point of a state trajectory. As observed by Bybee, Perkins, Pagliuca (1994) and Dahl (2000a, 200ob), grams developing along the same universal paths - although they might have originated in different initial inputs - usually converge at the end of the evolutionary process. They tend to acquire the same (within the margin of error, cf. the approximation of the infinite data discussed above) state. Such a convergent geometrical area of different state trajectories is equivalent to the concept of the attractor. Thus, an attractor may be considered a long-term steady behaviour of realistic grammatical evolutionary processes. For instance, all original resultative grams tend to develop towards past tenses which, at later phases of evolution, are reduced to the function of a remote narrative past, only (e.g. the passé simple in French). However, since the state of a gram at the terminal moment of its development will never be identical to the states displayed by other grams controlled by the same path-law, the attractor in the state-space of the model will always appear as a set of highly similar - but not duplicate - states. Topologically, final states will develop closer and closer to a fictionalized terminal situation. In other words, although a given class of grams tends to converge into a typologically similar category, the states of such constructions at highly advanced phases of the development will never repeat themselves, due to sensitivity of initial conditions, error inflation and aperiodicity. They will, rather, converge towards a portion of the state-space, more and more confined on the idealized attractor. 
Consequently, the realistic development of grams can be imagined as being dense: the points which represent evolutionary terminal stages are dense on the attractor, so that any point in the state-space is theoretically approached closely by a trajectory. Because of the aperiodicity, the confinement is infinite and, hence, the attractor can be understood as being equivalent to a strange attractor, as is characteristic of chaotic systems (for a similar observation, see Bybee 2010: 198).

Universal paths, if they are regarded as laws concerning the extensions of meaning, are usually complex in the sense that a single deterministic path typically consists of multiple sub-trajectories leading to various outputs. This signifies that the evolutionary state-space model will display more than one strange attractor. It will yield various sets of confined ultimate states. In the case where there is more than one attractor, the state-space of a linguistic dynamic development will be divided into a determined number of basins of attraction that separate from each other at the bifurcation points. These points equal a change in the qualitative pattern of attractors. Consequently, the state-space will include all possible outcomes of a given dynamic process with all the potential attractors, basins, and bifurcations. Up to a certain moment, the trajectories symbolizing the evolution of grams departing from different initial conditions are bound within an error margin similar to that assumed in measuring the initial state of the formations in question. However, at a given time $t$ due to the modification in parameters controlling the system, the developmental processes (idealized as state trajectories) will diverge exponentially. They will head towards two distinct terminal states (attractors) leading, thus, to the formation of two independent grammatical constructions. The parameters may be viewed as a certain set of relevant properties of the system within which the grams evolve. This set can include any environmental features, such as the behaviours offered by any component of the larger system in which the evolution of the gram(s) under analysis is embedded.

I will explain this phenomenon by using the example of the anterior path. As explained previously, the anterior path is one of the three major developmental rules governing the semantic expansion of resultative constructions. The other two are the simultaneous path (resultative > stative > present, see Andrason 2011a, 2011b, 2013a, 2013b, 2014b) and the evidential path (resultative $>$ inferential $>$ referential $>$ epistemic modality, see Aikhenvald 2004). Consequently, one obtains three possible strange attractors in realistic evolutionary cases: past tense, present tense and epistemic mood. If one focuses on two predominant evolutionary drifts (viz. the anterior and simultaneous paths) the following can be observed. When employed in resultative and, later, present perfect morphologies, static predicates typically evolve in accordance with the simultaneous path, acquiring the senses of a stative and a present (cf. Maslov 1988; Bybee, Perkins, Pagliuca 1994; Andrason 2013a, 2013b, 2014b; see also Andrason, Locatell 2016). On the other hand, dynamic verbs regularly are driven by the rule encapsulated by the anterior path, thus heading towards the values of a present perfect, a perfective and a simple past. Consequently, with respect to grams controlled by the resultative path, the difference in initial conditions (i.e. the fact of being a dynamic or a non-dynamic predicate) activates a higher 
predisposition for a certain path (either the anterior or the simultaneous path, respectively). At a determined point, where an important change in parameters occurs, this predisposition for distinct dynamic rules may lead to a split of the original resultative construction into two separate categories and, thus, to a new arrangement of the structure of the language. This split (or, more properly, bifurcation) may clearly be observed in Germanic languages, where the preterite-present verbs have entirely lost any functional or semantic relationship to dynamic verbs of the same morphology, even though both sets of constructions historically have their sources in the same gram, i.e. a resultative (stative) proper. Nowadays, the preterite-present verbs behave as a present tense (e.g. Icelandic pú kannt 'you know' and við kunnum 'we know') and are considered as a subtype of the paradigmatic Present. However, the latter (i.e. all the non-preterite-present verbs) constitute a subcategory of the paradigmatic Past (e.g. pú rannt 'you slipped' and við runnum 'we slipped', see Andrason 2013, 2014b; see also Birkmann 1987; Bybee, Perkins, Pagliuca 1994). The phenomenon of bifurcation of a single initial construction into two or more formations due to distinct initial conditions (which lead to acquisition of senses located on different paths) and due to the modification of parameters (i.e. the change in the evolving system or its subparts) is well-attested in the development of the Proto-Semitic form qatal(a) into two sister formations: qatal (perfect, perfective, past) and weqatal (future, modal) in Biblical Hebrew (Andrason 2011a, 2011b, 2012, 2013a; for a similar behaviour of the perfect in Ancient and Koine Greek, see Andrason, Locatell 2016).

The above-proposed model of the realistic evolution of grams fulfils the definition of chaos in non-mathematical theories (such as linguistics) as formulated in the second paper of the series. Consequently, the system which it represents can be viewed as chaotic. In particular, its fictionalized and idealized representation delivers a dynamic, non-linear, a-periodic structure which, although governed by deterministic rules or "equations" (encapsulated by paths in Path Theory), is (as far as long-term estimations for a concrete trajectory) unpredictable due to a high sensitivity to initial conditions. Topologically, it delivers bifurcations, basins and attractors, and, furthermore, exhibits a stretching-and-folding behaviour on these last ones. This stretching-and-folding character implies two things: an infinitively confined dense convergence of trajectories belonging to the same basin on the strange attractor and an exponential divergence of trajectories heading towards different attractors.

\section{Conclusion}

The present research has demonstrated that by applying the narrative of Chaos Theory, it is possible to view classical paths as universal, and harmonize this deterministic assumption with the unpredictability of concrete (especially long-term) grammatical evolutions.

Traditional paths posited by Path Theory should be viewed as abstract deterministic laws which specify the order of senses incorporated into the semantic potential 
of grams of a certain taxonomical class. These laws are universal and deterministic in the sense that they control the sequence of values gradually integrated into the semantic potential of a gram. The universality and the determinism of paths appear because we treat these paths in isolation from the environment, that is without taking into account the noise produced by other elements of the system. ${ }^{3}$ Although classical paths govern realistic developments, constituting idealized and fictionalized rules that show the direction of change, they are unable to represent actual and realistic evolutionary processes. Nevertheless, paths can be employed to model the realistic synchronic state of a gram at a certain time $t$, thus providing a map of the meaning of this form. The suitability of the paths for the synchronic mapping and for the representation of synchronic states makes it possible to employ them as the foundation of a state-space. A state-space is the most accurate manner (if not, the only accurate manner) of representing the evolution of realistic dynamic systems such as language. To be precise, the concrete evolution of a gram is portrayed as a trajectory of states - i.e. as a sequence of points where each point stands for a map built around a classical path. The trajectory is, thus, a collection of states acquired by a gram over a period of time. It shows how the semantic potential of a construction, mapped by means of a path, evolves. Consequently, a model of realistic evolutions of formations of a given taxonomical type (i.e. a crosslinguistic macro-evolutionary category) equals a state-space of all possible evolutions that a similar type of inputs may undergo, from those that are more likely to those that are less probable. It encompasses all possible trajectories such constructions can travel or all possible points of the space they can visit.

The state-space model of realistic evolutionary processes behaves as a prototypical chaotic system. In particular, it complies to the definition of chaos, applied to linguistics: the modelled system appears as dynamic, non-linear, a-periodic and long-term unpredictable despite being controlled by deterministic rules. As in chaotic systems, this unpredictability stems from a high sensitivity to initial conditions and non-linearity of interactions. Additionally, the model exhibits other exemplary features of chaos: strange attractors, basins, bifurcations and a stretching-and-folding behaviour of idealized trajectories.

As a result - and in agreement with Chaos Theory - the dissonance between the determinism of traditional paths, on the one hand, and the unpredictability of concrete evolutionary cases, on the other, can be overcome. Although laws are deterministic, an exact and complete outline of long-term evolution is unpredictable. The determinism-unpredictability paradox can be solved because the clines hypothesized in the two models do not represent the same phenomena. In Path Theory, paths are abstract rules and symbolize an arrangement of consecutively developed and incorporated meanings. In the chaotic model, trajectories refer to consecutive states of grams and depict concrete evolutionary processes. The unpredictability of realistic trajectories emerges not because of the non-deterministic

This deterministic view of traditional grammaticalization clines, which emerges in the chaotic model, significantly improves their epistemological status. 
character of grammaticalization paths, but due to the sensitivity to initial conditions and the non-linear inflation of an error margin. Accordingly, the chaos framework does not weaken the strength of paths, and neither does it eliminate the universal principles of grammaticalization. Quite to the contrary, it incorporates the findings of these approaches into a stronger model, treating them as abstract dynamic, deterministic equations.

Although this paper has shown the usefulness of the narrative of chaos for the study of language evolution, it has not exhausted the entire potential of Chaos Theory. Most importantly, it has referred to the narrative of chaos without using any topological analysis formulated on mathematical grounds. The model is purely narrative in contrast to genuine chaotic models which are numerical and have a geometric interpretation. This means that neither trajectories of realistic developments nor their state-space have been designed. The construction of such precise geometric representations is necessary for discovering higher generalizations. These generalizations, which typically emerge from state-spaces found in chaotic models, profoundly regularize realistic evolutions. The formulation of such a topological representation (which would transform the narrative of chaos into a more sophisticated, precise and numerical model) will constitute one of the main research activities of the author in the near future.

Lastly, one should bear in mind the following: even though chaos can be used to elucidate certain linguistic phenomena and even though numerically determined, computer simulated models of chaos do exist, a full application of Chaos Theory to linguistics may be problematic (Kretzschmar 2015). This has its roots in that the determination of the initial conditions for language evolution on a necessary level of precision is not yet possible. Perhaps, it will never be possible. Much of further research hinges on establishing these (workable) sets of precise initial conditions. Consequently, although the development of the chaotic systems is all theoretically possible, the practicalities of the use of such modelling in language-specific context may not be fully productive. The system may always require reference to a map of possible trajectories that is larger than that formulated for its local situation. Currently, complex dynamic neural network models are regarded more likely to account for complexities and variability of real-world systems, including language. The author of the present paper himself has commonly used complex models (and not necessarily chaotic models) to represent linguistic systems (Andrason 2016a). ${ }^{4}$

\section{References}

Aikhenvald A. 2004. Evidentiality. Oxford.

Andrason A. 2011a. The Biblical Hebrew verbal system in light of grammaticalization - the second generation. - Hebrew Studies 52: 19-53.

Andrason A. 2011b. The Biblical Hebrew Wayyiqtol - a dynamic definition. - Journal of Hebrew Scriptures 11.7: 1-50.

4 These important matters discussed in the last paragraph were suggested to me by an anonymous reviewer. 
Andrason A. 2012. Thermodynamic model of the Biblical Hebrew verbal system. - Eades D. (ed.). Grammaticalisation in Semitic. Journal of Semitic Studies Supplement Series 29. Oxford: 146-163.

Andrason A. 2013a. El sistema verbal hebreo en su contexto semítico: una visión dinámica. Estella. Andrason A. 2013b. The map of the meaning of the be riy formation in Basse Mandinka. Journal of West African Languages 40.2: 101-133.

Andrason A. 2014a. Qotel and its dynamics. [Part 2]. - Folia Orientalia 51: 139-153.

Andrason A. 2014b. From resultatives to present tenses: Simultaneous path of resultative formations. - Italian Journal of Linguistics 26: 1-58.

Andrason A. 2016a. A complex system of complex predicates: Tense, taxis, aspect and mood in Basse Mandinka from a grammaticalization and cognitive perspective. [PhD Diss., University of Stellenbosch].

Andrason A., Visser M. 2015. Affordances perspective and grammaticalization - incorporation of language, environment and users in the model of semantic paths. - Studies in Second Language Learning and Teaching 5.4: 663-698.

Andrason A. 2016b. From vectors to waves and streams. Semantic maps - a new generation. - Stellenbosch Papers in Linguistics 45: 1-29.

Andrason A., Locatell C. 2016. The perfect wave. - Biblical and Ancient Greek Linguistics 5: $7-121$.

Auyang S. 1998a. How science comprehends chaos. [Paper presented at the Department of the History of Science Harvard University. February 23, 1998; www.creatingtechnology. org/essays/chaos.htm].

Auyang S. 1998b. Foundations of complex-system theories. Cambridge.

Auyang S. 2000. Mind in everyday life and cognitive science. Cambridge.

Birkmann T. 1987. Präteritopräsentia. Tübingen.

Bishop R. 2011. Metaphysical and epistemological issues in complex systems. - Hooker C. (ed.). Philosophy of complex systems. Amsterdam: 105-136.

Bybee J., Perkins R., Pagliuca W. 1994. The evolution of grammar. Chicago.

Bybee J. 2010. Language, usage and cognition. Cambridge.

Cilliers P. et al. 2013. Complexity, modeling, and natural resource management. - Ecology and Society 18.3: 1-12.

Dahl Ö. 2000a. The tense and aspect systems of European languages in a typological perspective. - Dahl Ö. (ed.). Tense and aspect in the languages of Europe. Berlin: 3-25.

Dahl Ö. (ed.). 2000b. Tense and aspect in the languages of Europe. Berlin.

Devaney R. 1989. An introduction to chaotic dynamical systems. Redwood City.

Evans V., Green M. 2006. Cognitive Linguistics: An introduction. Edinburgh.

Fischer K. 2010. Quantitative methods in cognitive semantics. Introduction to the volume.Glyn D., Fischer K. (eds.). Quantiative methods in cognitive semantics: Corpus-driven approaches. Berlin: 43-59.

Geeraerts D. 2010. Theories of lexical semantics. Oxford.

Gilquin G. 2006. The place of prototypicality in corpus linguistics. - Gries S., Stefanowitsch A. (eds.). Corpora in Cognitive Linguistics. Berlin: 159-191.

Glyn D. 2010. Corpus-driven cognitive semantics introduction to the field. - Glyn D., Fischer K. (eds.). Quantiativem methods in cognitive semantics: Corpus-driven approaches. Berlin: 1-41.

Grevisse M. 1975. Le bon usage. [10 ${ }^{\text {th }}$ edition]. Gembloux.

Gries S. 2006. Corpus-based methods and cognitive semantics. - Gries S., Stefanowitsch A. (eds.). Corpora in Cognitive Linguistics. Berlin: 57-99. 
Gries S., Divjak D. 2010. Quantitative approaches in usage-based cognitive semantics: Myths, erroneous assumptions, and a proposal. - Glyn D., Fischer K. (eds.). Quantiative methods in cognitive semantics: Corpus-driven approaches. Berlin: 333-353.

Gries S., Stefanowitsch A. (eds.). 2006. Corpora in Cognitive Linguistics. Berlin.

Kretzschmar W. 2015. Language and complex system. Cambridge.

Larsen-Freeman D. 1997. Chaos/complexity science and language acquisition. - Applied Linguistics 18: 141-165.

Maslov J. 1988. Resultative, perfect and aspect. - Nedjalkov V. (ed.). Typology of resultative constructions. Amsterdam, Philadelphia: 63-85.

Richardson K., Cilliers P., Lissack M. 2007. Complexity science: A 'Gray' science for the 'stuff in between'. - Cilliers P. (ed.). Thinking complexity. Mansfield: 25-35.

Smith P. 1998. Explaining chaos. Cambridge.

Strogatz S. 1994. Nonlinear dynamics and chaos: With applications to physics, biology, chemistry, and engineering. New York.

Wagensberg J. 2007. Ideas sobre la complejidad del mundo. Barcelona. 